Supplement of Hydrol. Earth Syst. Sci., 23, 3405-3421, 2019

https://doi.org/10.5194/hess-23-3405-2019-supplement

(C) Author(s) 2019. This work is distributed under

the Creative Commons Attribution 4.0 License.

(c) (1)
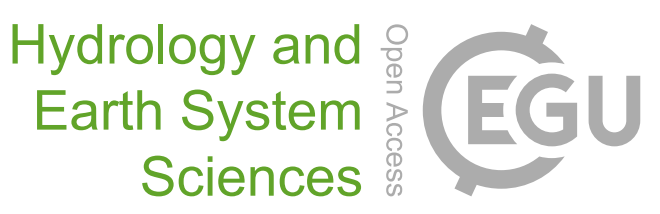

Supplement of

\title{
Improving hydrological projection performance under contrasting climatic conditions using spatial coherence through a hierarchical Bayesian regression framework
}

Zhengke Pan et al.

Correspondence to: Pan Liu (liupan@whu.edu.cn)

The copyright of individual parts of the supplement might differ from the CC BY 4.0 License. 
1 Supplement:

2 Table S1 The prior ranges of all unknown quantities in different scenarios

3 (1) Calibration in non-dry period and verification in dry period:

4 Scenario 1:

\begin{tabular}{ccccccccccccccccc}
\hline$\theta_{2-1}$ & $\theta_{2-1}$ & $\theta_{2-3}$ & $\mu_{2}$ & $\sigma_{3}$ & $\theta_{3-1}$ & $\theta_{4-1}$ & $\alpha_{1-1}$ & $\omega_{1-1}$ & $\theta_{3-2}$ & $\theta_{4-2}$ & $\alpha_{1-2}$ & $\omega_{1-2}$ & $\theta_{3-3}$ & $\theta_{4-3}$ & $\alpha_{1-3}$ & $\omega_{1-3}$ \\
\hline-10 & -10 & -10 & -100 & 0 & 0.1 & 1 & 1 & 0.0001 & 0.1 & 0.5 & 100 & 0.0001 & 0.1 & 0.1 & 1 & 0.0001 \\
10 & 10 & 10 & 100 & 6 & 200 & 10 & 600 & 0.4 & 300 & 20 & 1000 & 0.4 & 300 & 20 & 500 & 0.4 \\
\hline
\end{tabular}

6 Scenario 2:

\begin{tabular}{lllllllllllllllll}
\hline$\theta_{2-1}$ & $\theta_{2-1}$ & $\theta_{2-3}$ & $\mu_{3}$ & $\sigma_{3}$ & $\theta_{3-1}$ & $\theta_{4-1}$ & $\alpha_{1-1}$ & $\beta_{1-1}$ & $\theta_{3-2}$ & $\theta_{4-2}$ & $\alpha_{1-2}$ & $\beta_{1-2}$ & $\theta_{3-3}$ & $\theta_{4-3}$ & $\alpha_{1-3}$ & $\beta_{1-3}$ \\
\hline-6 & -6 & -6 & -0.4 & 0 & 1 & 0.5 & 1 & -300 & 1 & 0.1 & 100 & -300 & 0.1 & 2 & 1 & -200 \\
-6 & -6 & -6 & 0.4 & 0.1 & 500 & 10 & 600 & 300 & 300 & 20 & 600 & 500 & 400 & 20 & 800 & 300 \\
\hline
\end{tabular}

7

8 Scenario 3:

\begin{tabular}{llllllllllllllll}
\hline$\theta_{2-1}$ & $\theta_{2-1}$ & $\theta_{2-3}$ & $\mu_{2}$ & $\sigma_{2}$ & $\mu_{3}$ & $\sigma_{3}$ & $\theta_{3-1}$ & $\theta_{4-1}$ & $\alpha_{1-1}$ & $\theta_{3-2}$ & $\theta_{4-2}$ & $\alpha_{1-2}$ & $\theta_{3-3}$ & $\theta_{4-3}$ & $\alpha_{1-3}$ \\
\hline-5 & -5 & -5 & -200 & 0 & -0 & 0 & 1 & 0.5 & 1 & 1 & 0.1 & 100 & 1 & 0.5 & 100 \\
5 & 5 & 5 & 100 & 8 & 0.4 & 0.1 & 120 & 10 & 500 & 300 & 20 & 500 & 250 & 20 & 600 \\
\hline
\end{tabular}

10 Scenario 4:

\begin{tabular}{llllllllllllllllll}
\hline$\theta_{2-1}$ & $\theta_{3-1}$ & $\theta_{4-1}$ & $\alpha_{1-1}$ & $\beta_{1-1}$ & $\omega_{1-1}$ & $\theta_{2-2}$ & $\theta_{3-2}$ & $\theta_{4-2}$ & $\alpha_{1-2}$ & $\beta_{1-2}$ & $\omega_{1-2}$ & $\theta_{2-3}$ & $\theta_{3-3}$ & $\theta_{4-3}$ & $\alpha_{1-3}$ & $\beta_{1-3}$ & $\omega_{1-3}$ \\
\hline-10 & 1 & 0.1 & 1 & -300 & 0.0001 & -10 & 1 & 0.1 & 0 & -300 & 0 & -10 & 1 & 0.1 & 0 & -300 & 0.0001 \\
10 & 500 & 10 & 800 & 300 & 0.4 & 10 & 500 & 10 & 800 & 300 & 0.4 & 10 & 500 & 10 & 800 & 300 & 0.4 \\
\hline
\end{tabular}


(2) Calibration in dry period and verification in dry period:

14 Scenario 1:

\begin{tabular}{lllllllllllllllll}
\hline$\theta_{2-1}$ & $\theta_{2-2}$ & $\theta_{2-3}$ & $\mu_{2}$ & $\sigma_{2}$ & $\theta_{3-1}$ & $\theta_{4-1}$ & $\alpha_{1-1}$ & $\omega_{1-1}$ & $\theta_{3-2}$ & $\theta_{4-2}$ & $\alpha_{1-2}$ & $\omega_{1-2}$ & $\theta_{3-3}$ & $\theta_{4-3}$ & $\alpha_{1-3}$ & $\omega_{1-3}$ \\
\hline-10 & -10 & -10 & -60 & 0 & 1 & 0.5 & 1 & 0 & 1 & 0.5 & 1 & 0 & 1 & 0.1 & 1 & 0 \\
10 & 10 & 10 & 60 & 6 & 300 & 10 & 600 & 0.4 & 300 & 20 & 600 & 0.4 & 300 & 15 & 600 & 0.4 \\
\hline
\end{tabular}

16 Scenario 2:

\begin{tabular}{ccccccccccccccccc}
\hline$\theta_{2-1}$ & $\theta_{2-2}$ & $\theta_{2-3}$ & $\mu_{3}$ & $\sigma_{3}$ & $\theta_{3-1}$ & $\theta_{4-1}$ & $\alpha_{1-1}$ & $\beta_{1-1}$ & $\theta_{3-2}$ & $\theta_{4-2}$ & $\alpha_{1-2}$ & $\beta_{1-2}$ & $\theta_{3-3}$ & $\theta_{4-3}$ & $\alpha_{1-3}$ & $\beta_{1-3}$ \\
\hline-10 & -10 & -10 & 0.0001 & 0 & 1 & 0.5 & 1 & -300 & 1 & 0.1 & 1 & -400 & 0.1 & 0.5 & 1 & -400 \\
10 & 10 & 10 & 0.4 & 0.1 & 200 & 15 & 500 & 400 & 300 & 20 & 600 & 500 & 140 & 20 & 600 & 400 \\
\hline
\end{tabular}

Scenario 3:

\begin{tabular}{llllllllllllllll}
\hline$\theta_{2-1}$ & $\theta_{2-2}$ & $\theta_{2-3}$ & $\mu_{2}$ & $\sigma_{2}$ & $\mu_{3}$ & $\sigma_{3}$ & $\theta_{3-1}$ & $\theta_{4-1}$ & $\alpha_{1-1}$ & $\theta_{3-2}$ & $\theta_{4-2}$ & $\alpha_{1-2}$ & $\theta_{3-3}$ & $\theta_{4-3}$ & $\alpha_{1-3}$ \\
\hline-10 & -10 & -10 & -80 & 0 & 0 & 0 & 1 & 0.5 & 1 & 1 & 0.1 & 1 & 1 & 0.1 & 1 \\
10 & 10 & 10 & 80 & 6 & 0 & 0.1 & 200 & 10 & 500 & 400 & 20 & 600 & 400 & 20 & 600 \\
\hline
\end{tabular}

Scenario 4:

\begin{tabular}{llllllllllllllllll}
\hline$\theta_{2-1}$ & $\theta_{3-1}$ & $\theta_{4-1}$ & $\alpha_{1-1}$ & $\beta_{1-1}$ & $\omega_{1-1}$ & $\theta_{2-2}$ & $\theta_{3-2}$ & $\theta_{4-2}$ & $\alpha_{1-2}$ & $\beta_{1-2}$ & $\omega_{1-2}$ & $\theta_{2-3}$ & $\theta_{3-3}$ & $\theta_{4-3}$ & $\alpha_{1-3}$ & $\beta_{1-3}$ & $\omega_{1-3}$ \\
\hline-10 & 1 & 0.1 & 1 & -300 & 0.0001 & -10 & 1 & 0.1 & 1 & -300 & 0 & -10 & 1 & 0.1 & 1 & -300 & 0 \\
10 & 500 & 10 & 800 & 300 & 0.4 & 10 & 500 & 10 & 800 & 300 & 0.4 & 10 & 500 & 10 & 800 & 300 & 0.4 \\
\hline
\end{tabular}

Notes:

$\theta_{2-1}, \theta_{2-2}$ and $\theta_{2-3}$ refers to model parameter $\theta_{2}$ in catchment 225219,405219 and 405264 , respectively; $\theta_{3-1}, \theta_{3-2}$ and $\theta_{3-3}$ refer to model parameter $\theta_{3}$ in catchment 225219,405219 and 405264 , respectively; $\theta_{4-1}, \theta_{4-2}$ and $\theta_{4-3}$ refers to model parameter $\theta_{4}$ in catchment 225219,405219 and 405264 , respectively; $\mu_{2}, \sigma_{2}, \mu_{3}$ and $\sigma_{3}$ represent four hyper-parameters; $\alpha_{1-1}, \alpha_{1-2}$ and $\alpha_{1-3}$ refer to regression parameter $\alpha$ in catchment 225219,405219 and 
405264, respectively; $\beta_{1-1}, \beta_{1-2}$ and $\beta_{1-3}$ refer to regression parameter $\beta$ in catchment 225219,405219 and 405264 , respectively; $\omega_{1-1}, \omega_{1-2}$ and $\omega_{1-3}$ 27 refer to regression parameter $\omega$ in catchment 225219, 405219 and 405264, respectively. 